\title{
Time domain BEM for sound radiation of tires
}

\author{
Lothar Banz $^{1}$ • Heiko Gimperlein ${ }^{2,3}$ - Zouhair Nezhi ${ }^{4}$ - Ernst P. Stephan ${ }^{4}$
}

\begin{abstract}
This work investigates a time domain boundary element method for the acoustic wave equation in an exterior domain in the half-space $\mathbb{R}_{+}^{3}$. The Neumann problem is formulated as a boundary integral equation of the second kind, and the convergence and stability of conforming Galerkin approximations is studied in the complex geometry of a car or truck tire above a street. After a validation experiment, numerical results are presented in time or frequency domain for realistic benchmarks in traffic noise: the sound emission of vibrating tires, noise amplification in the horn-like geometry between the tire and the road, as well as the Doppler effect of a moving tire. The results are compared with calculations in frequency domain.
\end{abstract}

Keywords Time domain BEM - Sound radiation .

Horn effect · Computational acoustics · Boundary elements

Heiko Gimperlein

h.gimperlein@hw.ac.uk

Lothar Banz

lothar.banz@sbg.ac.at

Zouhair Nezhi

nezhi@ifam.uni-hannover.de

Ernst P. Stephan

stephan@ifam.uni-hannover.de

1 Department of Mathematics, University of Salzburg, Hellbrunner Straße 34, 5020 Salzburg, Austria

2 Maxwell Institute for Mathematical Sciences and Department of Mathematics, Heriot-Watt University, Edinburgh EH14 4AS, UK

3 Institut für Mathematik, Universität Paderborn, Warburger Str. 100, 33098 Paderborn, Germany

4 Institute of Applied Mathematics, Leibniz University Hannover, 30167 Hannover, Germany

\section{Introduction}

Efficient and accurate computational methods to simulate sound emission in space and time are of interest from modeling environmental noise to the acoustics of concert halls. This work investigates a time-domain Galerkin boundary element method for acoustic wave problems. The approach proves efficient, stable and accurate for long-time computations, and it is competitive with frequency domain methods for realworld sound emission of tires.

Time-domain boundary integral formulations provide a natural formulation for emission problems: as integral equations based on an exact fundamental solution they only discretize the boundary condition and are free of dispersion, allowing simulations for long-time. For traffic noise, the fully time dependent description is not only necessary to model the continuous sequence of acceleration, breaking and dynamic contact with the road, but after a Fourier transform in time may also be used to obtain frequency domain information for a broad range of frequencies. In particular, the time domain approach will be of interest to truly transient problems, which involve many frequencies, as well as to nonlinear problems that cannot be reformulated as a PDE in frequency domain, e.g. dynamic contact beyond the quasistatic approximation.

To be specific, this article considers the radiation of timedependent acoustic waves outside a bounded domain in the half-space above a rigid surface, e.g. a tire above the street, described by the Neumann problem

$$
\begin{aligned}
& \frac{1}{c^{2}} \frac{\partial^{2} p}{\partial \tau^{2}}-\Delta p=0 \text { in } \mathbb{R}^{+} \times \Omega^{e} \\
& p(0, x)=\frac{\partial p}{\partial \tau}(0, x)=0 \text { in } \Omega^{e} \\
& \frac{\partial p}{\partial n}(\tau, x)=-\rho \frac{\partial^{2} u_{n}}{\partial \tau^{2}}(\tau, x)-\frac{\partial p^{I}}{\partial n}(\tau, x) \text { in } \mathbb{R}^{+} \times \Gamma
\end{aligned}
$$




$$
\frac{\partial p}{\partial x_{3}}(\tau, x)=0 \text { in } \mathbb{R}^{+} \times \Gamma_{\infty}
$$

Here, $\tau$ denotes the time, $c$ the speed of sound and $\rho$ the density of air. The Lipschitz domain $\Omega^{e}$ is given by the halfspace $\mathbb{R}_{+}^{3}$ without the tire, $\Gamma$ is the surface of the tire in $\mathbb{R}_{+}^{3}$ and $\Gamma_{\infty}$ the plane $\left\{x \in \mathbb{R}^{3}: x_{3}=0\right\}$ outside the tire. The emitted sound pressure is denoted by $p(\tau, x)$, and $p^{I}(\tau, x)$ describes an incident wave. The coupling of the tire vibrations and sound pressure is given by the boundary condition (1c), where $u_{n}$ describes the displacement of the tire in the outer normal direction.

Note that the set-up includes both a tire which rests on the street as well as an elevated tire.

The article discusses the numerical approximation of this boundary problem based on a time-domain integral formulation of the second kind and its Galerkin discretization. Numerical experiments study the sound emission of tires at frequencies up to $2000 \mathrm{~Hz}$, based on data and models obtained within the project "LeiStra3" of the German highway authorities. Compared to earlier works like [1], this article demonstrates the convergence and relevance of the considered Galerkin method for large, real-world problems in acoustics and for the singular horn geometry of the halfspace. The time domain BEM is validated and compared with Burton-Miller stabilized frequency domain formulations for some realistic benchmarks in traffic noise. Results for moving or rolling sound sources indicate the opportunities of time-domain computations.

One expects similar results for the radiation of waves in other contexts, and for integral formulations of the first kind. Future work will explore the behavior of the approach at higher frequencies and for nonlinear, dynamic contact, the coupling to finite element models for the tire dynamics and the use of adaptive methods.

Time domain boundary integral formulations for hyperbolic equations and their numerical solution go back to Friedman and Shaw [2], resp. Cruse and Rizzo [3]. Mansur [4] then developed one of the first modern boundary element methods, and many of the basic algorithmic observations go back to him. A first mathematical analysis of time dependent Galerkin boundary element methods was then done in a seminal work by Bamberger and Ha-Duong [5]. Numerical experiments for integral equations of the second kind in the full space were reported by Ding, Forestier and Ha-Duong [1], and the actual realization of the numerical marching-onin-time scheme include the Ph.D. thesis of Terrasse [6] as well as [7]. The discretization and accurate computation of the entries in the Galerkin matrix elements has been considered in detail by Maischak, Ostermann and Stephan [8,9], and one may refer to the dissertation of Ostermann [10] for further algorithmic details. As an alternative to time domain
BEM, one can mention the recent progress for convolution quadrature methods $[11,12]$.

In the special case of the half-space, the current work is motivated by the recent exact formulas obtained by Ochmann [13], as they allow acoustic Robin boundary conditions on the surface of the street. Stable time domain boundary elements for acoustics have recently been considered in $[14,15]$. For engineering applications such as tire noise, also wave propagation in moving coordinate systems or with moving sources proves crucial. Refer to [16-18] for some previous works. Truly transient phenomena that cannot be simulated in the frequency domain involve contact and damage, see $[19,20]$ for time domain BEM approaches to such problems.

Future work to couple the acoustics with the tire dynamics will rely on the recent advances in time dependent coupling of finite and boundary element methods [21]. Also fast multipole methods will be of interest, [22] and [23].

An overview of the mathematical background can be found in [24], [25] and [26], see also [27] for the case of the half-space.

Concerning the structure of this article: After recalling the formulation of (1) as a time-domain boundary integral equation in Sect. 2, the article presents the Galerkin discretization of a weak formulation in Sect. 3. The following section discusses algorithmic considerations in the computation of the matrix elements as well as the resulting marching-on-in-time scheme. After validating our boundary element method in an experiment on the sphere (Sect. 5), the remaining sections present numerical experiments for the sound emission in relevant engineering problems: The noise of vibrating car and truck tires in frequency domain, as perceived by a human ear; the noise amplification in the horn-like geometry where a car tire meets the road; and the noise emitted by moving and rolling tires, in which the computations e.g. resolve a relative Doppler shift of $2 \%$ of the emitted frequency.

\section{Time domain BEM for sound radiation}

It proves useful to perform computations in units adapted to $c \approx 343 \mathrm{~ms}^{-1}$ : scaling the time variable, $t=c \tau$, (1) becomes

$$
\begin{aligned}
& \frac{\partial^{2} p}{\partial t^{2}}-\Delta p=0 \text { in } \mathbb{R}^{+} \times \Omega^{e} \\
& p(0, x)=\frac{\partial p}{\partial t}(0, x)=0 \text { in } \Omega^{e} \\
& \frac{\partial p}{\partial n}(t, x)=-\rho c^{2} \frac{\partial^{2} u_{n}}{\partial t^{2}}(t, x)-\frac{\partial p^{I}}{\partial n}(t, x) \text { in } \mathbb{R}^{+} \times \Gamma
\end{aligned}
$$

$\frac{\partial p}{\partial x_{3}}(t, x)=0$ in $\mathbb{R}^{+} \times \Gamma_{\infty}$. 
As the spatial domain $\Omega^{e}$ is unbounded, it is advantageous to transform the boundary value problem into a boundary integral equation. To do so, one makes a single layer potential ansatz for the sound pressure [25]

$$
\begin{aligned}
p(t, x)=S \varphi(t, x)= & \frac{1}{4 \pi} \int_{\Gamma} \frac{\varphi(t-|x-y|, y)}{|x-y|} d s_{y} \\
& +\frac{1}{4 \pi} \int_{\Gamma} \frac{\varphi\left(t-\left|x-y^{\prime}\right|, y\right)}{\left|x-y^{\prime}\right|} d s_{y},
\end{aligned}
$$

where $\varphi(s, y)=0$ for $s \leq 0$ and $y^{\prime}$ is the mirror image of $y$ with respect to the plane $\Gamma_{\infty}$. Substituting (3) into the wave equation, (2a), (2b) and (2d) are automatically satisfied. With the jump relation of the adjoint double layer potential, Equation (2c) leads to an equivalent boundary integral equation of second kind for the unknown density $\varphi(t, x)$ on $\Gamma$ [25]:

$$
\begin{aligned}
& \left(-I+K^{\prime}\right) \varphi(t, x)=2 \frac{\partial p}{\partial n}(t, x)=-2 \rho c^{2} \frac{\partial^{2} u_{n}}{\partial t^{2}}(t, x) \\
& -2 \frac{\partial p^{I}}{\partial n}(t, x) .
\end{aligned}
$$

Here, the transient adjoint double layer potential $K^{\prime}$ for the half-space is (with $\dot{\varphi}=\partial_{t} \varphi$ )

$$
\begin{aligned}
K^{\prime} \varphi(t, x)= & \frac{1}{2 \pi} \int_{\Gamma} \frac{n_{x}^{\top}(y-x)}{|x-y|} \\
& \times\left(\frac{\varphi(t-|x-y|, y)}{|x-y|^{2}}+\frac{\dot{\varphi}(t-|x-y|, y)}{|x-y|}\right) d s_{y} \\
& +\frac{1}{2 \pi} \int_{\Gamma} \frac{n_{x}^{\top}\left(y^{\prime}-x\right)}{\left|x-y^{\prime}\right|} \\
& \times\left(\frac{\varphi\left(t-\left|x-y^{\prime}\right|, y\right)}{\left|x-y^{\prime}\right|^{2}}+\frac{\dot{\varphi}\left(t-\left|x-y^{\prime}\right|, y\right)}{\left|x-y^{\prime}\right|}\right) d s_{y} .
\end{aligned}
$$

Partially absorbing, acoustic boundary conditions on the road can be treated by a modification of $K^{\prime}$, see $[13,27]$.

This article investigates a Galerkin method to solve (4) approximately. Its efficiency is demonstrated in benchmark computations for the sound radiation of tires.

\section{Boundary integral formulation and its discretization}

Compared to the elliptic case, more care is needed to interpret the variational formulation of (4), and space-time anisotropic Sobolev spaces provide a convenient language. Let $\sigma>0$, and assume that boundary of the surface $\Gamma$ is empty. The space $H_{\sigma}^{s}\left([0, \infty), H^{r}(\Gamma)\right)$ consists of certain distributions $\phi$ on $[0, \infty) \times \Gamma$, vanishing at $t=0$, such that in local coordinates the space-time Fourier-Laplace transform $\mathcal{F} \phi$ satisfies

$\|\phi\|_{s, r, \Gamma}=\left(\iint|\omega+i \sigma|^{2 s}\left(|\omega+i \sigma|^{2}+|\xi|^{2}\right)^{r}\right.$

$$
\left.\times|\mathcal{F} \phi(\omega+i \sigma, \xi)|^{2} d \xi d \omega\right)^{\frac{1}{2}}<\infty .
$$

In these spaces, the operator $-I+K^{\prime}$ maps the space $H_{\sigma}^{s}\left([0, \infty), H^{-\frac{1}{2}}(\Gamma)\right)$ to $H_{\sigma}^{s-1}\left([0, \infty), H^{-\frac{1}{2}}(\Gamma)\right)$. The Neumann problem (4) is well-posed in the sense that for $\frac{\partial p}{\partial n} \in$ $H_{\sigma}^{s+2}\left([0, \infty), H^{-\frac{1}{2}}(\Gamma)\right)$ there exists a unique solution $\varphi \in$ $H_{\sigma}^{s}\left([0, \infty), H^{-\frac{1}{2}}(\Gamma)\right)$, and $\|\varphi\|_{s,-\frac{1}{2}, \Gamma} \leq C\left\|\partial_{n} p\right\|_{s+2,-\frac{1}{2}, \Gamma}$ for some constant $C$ independent of $\partial_{n} p$. See $[1,25,27,28]$ for related results, as well as the necessary modifications when $\partial \Gamma \neq \emptyset$.

The Eq. (4) will be discretized from its variational formulation:

Find $\varphi(t, x) \in H_{\sigma}^{\frac{1}{2}}\left([0, \infty), H^{-\frac{1}{2}}(\Gamma)\right)$ such that for all test functions $\psi(t, x) \in H_{\sigma}^{\frac{1}{2}}\left([0, \infty), H^{-\frac{1}{2}}(\Gamma)\right)$ there holds:

$$
\begin{aligned}
\int_{0}^{\infty} & e^{-2 \sigma t} \int_{\Gamma}\left(-I+K^{\prime}\right) \varphi(t, x) \psi(t, x) d s_{x} d t \\
& =2 \int_{0}^{\infty} e^{-2 \sigma t} \int_{\Gamma} \frac{\partial p}{\partial n}(t, x) \psi(t, x) d s_{x} d t
\end{aligned}
$$

By the above-mentioned properties of $-I+K^{\prime}$, it admits a unique solution when $\frac{\partial p}{\partial n} \in H_{\sigma}^{\frac{5}{2}}\left([0, \infty), H^{-\frac{1}{2}}(\Gamma)\right)$, i.e. for sufficiently smooth functions of time.

While $\sigma>0$ is required by the analysis, for numerical computations $\sigma=0$ will be used. Even for $\sigma>0$, however, and in contrast to both time-domain integral equations of the first kind and time-independent problems, the bilinear form above is not coercive, which may lead to instabilities.

For the construction of the boundary element spaces the surface $\Gamma$ is discretized into triangles $\Gamma_{i}$ satisfying the usual regularity constraints, as well as the time interval into subintervals $I_{m}=\left(t_{m-1}, t_{m}\right]$ of length $\Delta t$. The approximation $\varphi_{\Delta t, h}$ of the density $\varphi$ is then sought in

$X_{\Delta t, h}=\left\{v(t, x)=\sum_{m=1}^{N_{t}} \sum_{i=1}^{N_{s}} b_{i}^{m} \gamma^{m}(t) \varphi_{i}(x)\right\}$,

where $\varphi_{i}$ and $\gamma^{m}$ are piecewise constant basis functions with support on $\Gamma_{i}, I_{m}$, respectively. As test functions the basis functions of $X_{\Delta t, h}$ given by

$\psi_{\Delta t, h}(t, x)=\gamma^{n}(t) \varphi_{j}(x) \quad 1 \leq j \leq N_{s}, \quad 1 \leq n \leq N_{t}$

are used. The discretized formulation of (5) in $X_{\Delta t, h}$ reads: Find $\varphi_{\Delta t, h} \in X_{\Delta t, h}$ such that

$$
\begin{gathered}
\int_{0}^{T} \int_{\Gamma}\left(-I+K^{\prime}\right) \varphi_{\Delta t, h}(t, x) \gamma^{n}(t) \varphi_{j}(x) d s_{x} d t \\
\quad=2 \int_{0}^{T} \int_{\Gamma} \frac{\partial p}{\partial n}(t, x) \gamma^{n}(t) \varphi_{j}(x) d s_{x} d t
\end{gathered}
$$


for $1 \leq j \leq N_{s}, 1 \leq n \leq N_{t}$. While one obtains unique and stable solutions in practice, proving this remains a known and open problem.

\section{Algorithmic considerations}

Because of the tensor product of the space time discretization, the left hand side of (7) becomes

$$
\begin{aligned}
- & \sum_{m=1}^{N_{t}} \sum_{i=1}^{N_{s}} b_{i}^{m} \int_{\Gamma} \varphi_{i}(x) \varphi_{j}(x) d s_{x} \int_{0}^{T} \gamma^{m}(t) \gamma^{n}(t) d t \\
+ & \sum_{m=1}^{N_{t}} \sum_{i=1}^{N_{s}} b_{i}^{m}\left[\int_{\Gamma} \int_{\Gamma}\left(\int_{0}^{T} \dot{\gamma}^{m}(t-|x-y|) \gamma^{n}(t) d t\right)\right. \\
& \times \frac{n_{x}^{\top}(x-y)}{2 \pi|x-y|^{2}} \varphi_{i}(y) \varphi_{j}(x) d s_{x} d s_{y} \\
& +\int_{\Gamma} \int_{\Gamma}\left(\int_{0}^{T} \gamma^{m}(t-|x-y|) \gamma^{n}(t) d t\right) \\
& \times \frac{n_{x}^{\top}(x-y)}{2 \pi|x-y|^{3}} \varphi_{i}(y) \varphi_{j}(x) d s_{x} d s_{y} \\
+ & \int_{\Gamma} \int_{\Gamma}\left(\int_{0}^{T} \dot{\gamma}^{m}\left(t-\left|x-y^{\prime}\right|\right) \gamma^{n}(t) d t\right) \\
& \times \frac{n_{x}^{\top}\left(x-y^{\prime}\right)}{2 \pi\left|x-y^{\prime}\right|^{2}} \varphi_{i}(y) \varphi_{j}(x) d s_{x} d s_{y} \\
& +\int_{\Gamma} \int_{\Gamma}\left(\int_{0}^{T} \gamma^{m}\left(t-\left|x-y^{\prime}\right|\right) \gamma^{n}(t) d t\right) \\
& \left.\times \frac{n_{x}^{\top}\left(x-y^{\prime}\right)}{2 \pi\left|x-y^{\prime}\right|^{3}} \varphi_{i}(y) \varphi_{j}(x) d s_{x} d s_{y}\right] .
\end{aligned}
$$

That expression can be simplified significantly by noting that

$$
\begin{aligned}
& \int_{0}^{T} \gamma^{m}(t) \gamma^{n}(t) d t=\Delta t \delta_{n m}, \\
& \int_{0}^{T} \dot{\gamma}^{m}(t-|x-y|) \gamma^{n}(t) d t=-\chi_{E_{n-m-1}}(x, y)+\chi_{E_{n-m}}(x, y), \\
& \int_{0}^{T} \gamma^{m}(t-|x-y|) \gamma^{n}(t) d t=\left(t_{n-m+1}-|x-y|\right) \chi_{E_{n-m}}(x, y) \\
& \quad+\left(|x-y|-t_{n-m-1}\right) \chi_{E_{n-m-1}}(x, y),
\end{aligned}
$$

using that the derivative of $\gamma^{m}$ is a difference of Dirac deltadistributions in $t_{m-1}$, resp. $t_{m}$. Here the light cone $E_{l}$ is defined as

$$
E_{l}:=\left\{(x, y) \in \Gamma \times \Gamma: t_{l} \leq|x-y| \leq t_{l+1}\right\},
$$

and its indicator function $\chi_{E_{l}}(x, y)=1$ if $(x, y) \in E_{l}$ and $\chi_{E_{l}}(x, y)=0$ otherwise. Herewith, one obtains for (8)
$-\Delta t \sum_{i=1}^{N_{s}} b_{i}^{n} \int_{\Gamma} \varphi_{i}(x) \varphi_{j}(x) d s_{x}+\sum_{m=1}^{N_{t}} \sum_{i=1}^{N_{s}} b_{i}^{m}\left(A_{i m, j n}+A_{i m, j n}^{\prime}\right)$,

where

$$
\begin{aligned}
A_{i m, j n}:= & -\int_{E_{n-m-1}} \frac{n_{x}^{\top}(x-y)}{2 \pi|x-y|^{2}} \varphi_{i}(y) \varphi_{j}(x) d s_{x} d s_{y} \\
& +\int_{E_{n-m}} \frac{n_{x}^{\top}(x-y)}{2 \pi|x-y|^{2}} \varphi_{i}(y) \varphi_{j}(x) d s_{x} d s_{y} \\
& +t_{n-m+1} \int_{E_{n-m}} \frac{n_{x}^{\top}(x-y)}{2 \pi|x-y|^{3}} \varphi_{i}(y) \varphi_{j}(x) d s_{x} d s_{y} \\
& -\int_{E_{n-m}} \frac{n_{x}^{\top}(x-y)}{2 \pi|x-y|^{2}} \varphi_{i}(y) \varphi_{j}(x) d s_{x} d s_{y} \\
& +\int_{E_{n-m-1}} \frac{n_{x}^{\top}(x-y)}{2 \pi|x-y|^{2}} \varphi_{i}(y) \varphi_{j}(x) d s_{x} d s_{y} \\
& -t_{n-m-1} \int_{E_{n-m-1}} \frac{n_{x}^{\top}(x-y)}{2 \pi|x-y|^{3}} \varphi_{i}(y) \varphi_{j}(x) d s_{x} d s_{y} .
\end{aligned}
$$

$A_{i m, j n}^{\prime}$ is defined analogously by taking $y^{\prime}$ instead of $y$ and, hence, $E_{n-m}^{\prime}, E_{n-m-1}^{\prime}$ instead of $E_{n-m}, E_{n-m-1}$. Therefore, (8) becomes

$$
\begin{aligned}
- & \Delta t \sum_{i=1}^{N_{s}} b_{i}^{m} \int_{\Gamma} \varphi_{i}(x) \varphi_{j}(x) d s_{x} \\
& +\sum_{m=1}^{N_{t}} \sum_{i=1}^{N_{s}} b_{i}^{m}\left[t _ { n - m + 1 } \left(\int_{E_{n-m}} \frac{n_{x}^{\top}(x-y)}{2 \pi|x-y|^{3}} \varphi_{i}(y) \varphi_{j}(x) d s_{x} d s_{y}\right.\right. \\
& \left.+\int_{E_{n-m}^{\prime}} \frac{n_{x}^{\top}\left(x-y^{\prime}\right)}{2 \pi\left|x-y^{\prime}\right|^{3}} \varphi_{i}(y) \varphi_{j}(x) d s_{x} d s_{y}\right) \\
& -t_{n-m-1}\left(\int_{E_{n-m-1}} \frac{n_{x}^{\top}(x-y)}{2 \pi|x-y|^{3}} \varphi_{i}(y) \varphi_{j}(x) d s_{x} d s_{y}\right. \\
& \left.\left.+\int_{E_{n-m-1}^{\prime}} \frac{n_{x}^{\top}\left(x-y^{\prime}\right)}{2 \pi\left|x-y^{\prime}\right|^{3}} \varphi_{i}(y) \varphi_{j}(x) d s_{x} d s_{y}\right)\right]
\end{aligned}
$$

Hence, (8) can be written compactly in the matrix form

$-\Delta t \cdot M \varphi^{n}+\sum_{m=1}^{n}\left(K^{\prime}\right)^{n-m} \varphi^{m}$

with the vector $\varphi^{n}=\left(b_{1}^{n}, \ldots, b_{N_{s}}^{n}\right)^{\top}$, the mass matrix $M$ and

$$
\begin{aligned}
\left(K^{\prime}\right)^{l}:= & t_{l+1}\left(\int_{E_{l}} \frac{n_{x}^{\top}(x-y)}{2 \pi|x-y|^{3}} \varphi_{i}(y) \varphi_{j}(x) d s_{x} d s_{y}\right. \\
& \left.+\int_{E_{l}^{\prime}} \frac{n_{x}^{\top}\left(x-y^{\prime}\right)}{2 \pi\left|x-y^{\prime}\right|^{3}} \varphi_{i}(y) \varphi_{j}(x) d s_{x} d s_{y}\right)
\end{aligned}
$$




$$
\begin{aligned}
& -t_{l-1}\left(\int_{E_{l-1}} \frac{n_{x}^{\top}(x-y)}{2 \pi|x-y|^{3}} \varphi_{i}(y) \varphi_{j}(x) d s_{x} d s_{y}\right. \\
& \left.+\int_{E_{l-1}^{\prime}} \frac{n_{x}^{\top}\left(x-y^{\prime}\right)}{2 \pi\left|x-y^{\prime}\right|^{3}} \varphi_{i}(y) \varphi_{j}(x) d s_{x} d s_{y}\right) .
\end{aligned}
$$

For the right hand side $F^{n}$ in (7) one obtains

$$
\begin{aligned}
\int_{0}^{T} & \int_{\Gamma} \frac{\partial p}{\partial n}(t, x) \gamma^{n}(t) \varphi_{j}(x) d s_{x} d t \\
= & -\rho c^{2} \int_{\Gamma} \int_{t_{n-1}}^{t_{n}} \dot{v}_{n}(t, x) d t \varphi_{j}(x) d s_{x} \\
& -\int_{\Gamma} \int_{t_{n-1}}^{t_{n}} \frac{\partial p^{I}}{\partial n}(t, x) d t \varphi_{j}(x) d s_{x}
\end{aligned}
$$

with $v_{n}=\dot{u}_{n}$ the normal component of the particle velocity. Hence, $F^{n}=\left(f_{1}^{n}, \ldots, f_{N_{s}}^{n}\right)^{\top}$ with

$$
\begin{aligned}
f_{j}^{n}= & -\rho c^{2}\left[\int_{\Gamma} v_{n}\left(t_{n}, x\right) \varphi_{j}(x) d s_{x}-\int_{\Gamma} v_{n}\left(t_{n-1}, x\right) \varphi_{j}(x) d s_{x}\right] \\
& -\int_{\Gamma} \int_{t_{n-1}}^{t_{n}} \frac{\partial p^{I}}{\partial n}(t, x) d t \varphi_{j}(x) d s_{x} \quad 1 \leq j \leq N_{s} .
\end{aligned}
$$

The Galerkin discretization in space and time leads to a blocklower-triangular system of equations, which can be solved by blockwise forward substitution. The resulting so-called marching-on-in-time (MOT) scheme, Algorithm 1, see also $[10,29]$, is given by

$\left(-\Delta t \cdot M+\left(K^{\prime}\right)^{0}\right) \varphi^{n}=2 F^{n}-\sum_{m=1}^{n-1}\left(K^{\prime}\right)^{n-m} \varphi^{m}$,

which is solved in every time step. The above fully discrete systems involve the computation of a series of matrices, that are sparsely populated because the domain of integration $E_{l}$ within the light cone restricts the number of interacting elements per time step. Note that the computation of each matrix only depends on the time difference [10]. Furthermore (see [10, Sec. 2.3.2]) for bounded surfaces $\Gamma$ the matrices $\left(K^{\prime}\right)^{n-m}$ vanish whenever the time difference $l:=n-m$ satisfies

$l>\left[\frac{\operatorname{diam} \Gamma}{\Delta t}\right]$,

i.e. the light cone has traveled through the entire surface $\Gamma$. Hence, from then onwards, the MOT scheme only requires to compute the load vector, preform some matrix-vector multiplications as well as solving a system of linear equations.

The most CPU-time consuming contribution in the MOT algorithm is the computation of the matrix entries, even though the resulting matrices are sparse. See [8-10] for

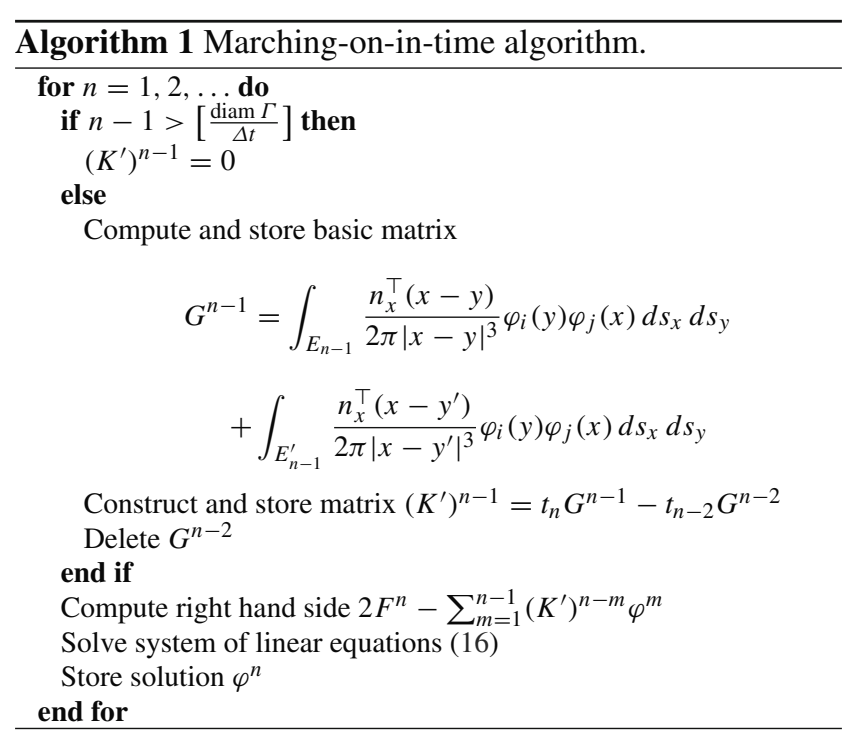

an efficient $h p$-composite Gauss-quadrature to compute the entries in $G^{l}$.

\section{Validation on a problem with known pressure and density}

To analyze the convergence properties of the discussed method, first a wave problem with known solution $p$ is considered in the exterior of a unit ball in $\mathbb{R}^{3}$. As the Laplacian of a radial function $u(r)$ in $\mathbb{R}^{3}$ is given by

$\Delta u=\frac{1}{r}(r u)_{r r}=\left(\frac{\partial^{2}}{\partial r^{2}}+\frac{1}{r} \frac{\partial}{\partial r}\right) u$,

the wave equation (2a) in this case becomes

$$
\left(\frac{\partial^{2}}{\partial t^{2}}-\frac{\partial^{2}}{\partial r^{2}}-\frac{1}{r} \frac{\partial}{\partial r}\right) u(t, r)=0
$$

It admits the general solution

$u(t, r)=r^{-1}[\tilde{\phi}(r+t)+\tilde{\psi}(r-t)]$ with $r=|x|$,

where $\tilde{\phi}$ and $\tilde{\psi}$ are functions on $\mathbb{R}$ determined by the initial conditions [29, Sec. 5.2]. For the rigid half-space, where $\frac{\partial p}{\partial n}(t, x)=0$ when $x_{3}=0$, the solution takes the form

$p(t, x)=u(t, r(h))+u(t, r(-h))$,

with $r(h)=\left\|x_{1}, x_{2}, x_{3}-h-1\right\|_{2}$ and $r(-h)=\| x_{1}, x_{2}, x_{3}$ $+h+1 \|_{2}$ and where $h$ is the distance of the ball to the $x_{1} x_{2}-$ plane. Choosing the initial conditions $u(0, x)=u_{0}(|x|)$ and $\dot{u}(0, x)=0$ with 
$u_{0}(s)= \begin{cases}1+\cos \left(\frac{\pi s}{R}\right) & |s|<R \\ 0 & |s| \geq R\end{cases}$

for some fixed $0<R<1$, one obtains the solution

$$
\begin{aligned}
p(t, & x)=\frac{r(h)-t}{2 r(h)} u_{0}(r(h)-t)+\frac{r(-h)-t}{2 r(-h)} u_{0}(r(-h)-t) \\
= & \frac{r(h)-t}{2 r(h)}\left[1+\cos \left(\frac{\pi(r(h)-t)}{R}\right)\right] H(R-|r(h)-t|) \\
& +\frac{r(-h)-t}{2 r(-h)}\left[1+\cos \left(\frac{\pi(r(-h)-t)}{R}\right)\right] \\
& \times H(R-|r(-h)-t|) .
\end{aligned}
$$

Here, $H(t)$ denotes the Heaviside function. Note that $p(0, x)=0$ in the half-space outside the ball. A straightforward, somewhat lengthy calculation yields the corresponding right hand side [29]

$$
\begin{aligned}
f= & \partial_{n} p=\partial_{r} p \\
= & {\left[\frac{t}{2 r(h)^{2}}\left(1+\cos \left(\frac{\pi(r(h)-t)}{R}\right)\right)\right.} \\
& \left.-\frac{\pi}{R} \frac{r(h)-t}{2 r(h)} \sin \left(\frac{\pi(r(h)-t)}{R}\right)\right] \\
& \times H(R-|r(h)-t|) \\
& +\frac{x^{2}+y^{2}+z^{2}-(h+1)^{2}}{r(h) r(-h)}\left(\left[\frac{t}{2 r(-h)^{2}}\right.\right. \\
& \times\left(1+\cos \left(\frac{\pi(r(-h)-t)}{R}\right)\right) \\
& \left.-\frac{\pi}{R} \frac{r(-h)-t}{2 r(-h)} \sin \left(\frac{\pi(r(-h)-t)}{R}\right)\right] \\
& \times H(R-|r(-h)-t|)) .
\end{aligned}
$$

The density for the single layer potential ansatz can be calculated to be $[29,30]$

$\varphi(t, x)=-2 \sum_{k=0}^{\lfloor t / 2\rfloor} f_{1}(t-2 k)+2 \sum_{k=0}^{\lfloor t / 2\rfloor} \int_{2 k}^{t} e^{-(s-2 k)} f_{1}(t-s) d s$

where $f_{1}(t)$ equals

$$
\begin{gathered}
{\left[\frac{t}{2 r(h)^{2}}\left(1+\cos \left(\frac{\pi(r(h)-t)}{R}\right)\right)-\frac{\pi}{R} \frac{r(h)-t}{2 r(h)}\right.} \\
\left.\sin \left(\frac{\pi(r(h)-t)}{R}\right)\right] H(R-|r(h)-t|) .
\end{gathered}
$$

Figure 1 shows the good qualitative agreement of the analytic and approximate solution in $x_{0}=(0,0,2.8)^{\top}$ for $R=0.9, h=0.63, \Delta t=0.1$ and 1080 uniform triangles. Figure 2 shows the relative discretization errors

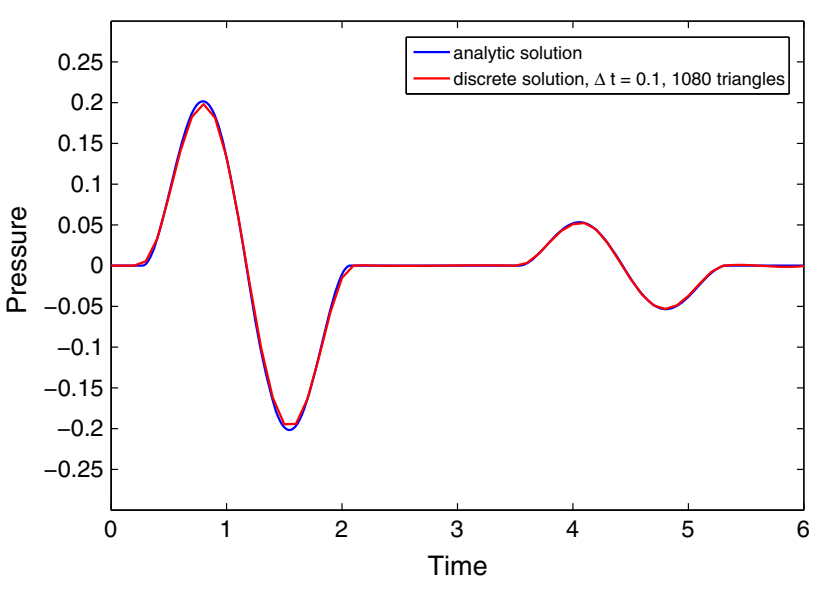

Fig. 1 Exact sound pressure and its Galerkin approximation in $x_{0}=$ $(0,0,2.8)$

$\frac{\left\|\varphi_{\Delta t, h}-\varphi\right\|_{L^{2}\left([0,10] ; L^{2}(\Gamma)\right)}}{\|\varphi\|_{L^{2}\left([0,10] ; L^{2}(\Gamma)\right)}}$ and $\frac{\left\|p_{\Delta t, h}\left(t, x_{0}\right)-p\left(t, x_{0}\right)\right\|_{L^{2}([0,10])}}{\left\|p\left(t, x_{0}\right)\right\|_{L^{2}([0,10])}}$

for a family of discrete solutions, where $\varphi_{\Delta t, h}$ is the TD-BE Galerkin approximation of $\varphi$ and $p_{\Delta t, h}=S \varphi_{\Delta t, h}$. It indicates a convergence rate of 0.4 for the density, resp. 0.65 for the sound pressure, with respect to the degrees of freedom (dof) given by the product of number of time steps and number of triangles. Note that one does expect a smaller convergence rate compared to a time-independent problem, due to the lack of ellipticity of the wave equation. For the numerical experiments, both the time step size $\Delta t$ as well as the mesh size $h$ have been halved four times starting from $\Delta t=2^{-1}$. The ratio of the mesh size $h$ and time step size $\Delta t$ is $\Delta t / h \approx 0.38$. See Fig. 3 for two corresponding spatial meshes.

Note that these experimental convergence results in the time domain imply convergence results in the frequency domain for the Fast Fourier Transform of the numerical solution, not at a single frequency, but in a weighted $L^{2}$-sense. Indeed, as the FFT preserves $L^{2}$-norms, the relative numerical $L^{2}$-errors of $\hat{\phi}_{\Delta t, h}(\omega, x)$ and $\hat{p}_{\Delta t, h}(\omega, x)$ coincide with the errors depicted in Fig. 2.

\section{Numerical experiments for a vibrating tire}

A steady-state rolling tire experiences periodic deformations, which enter our computations through the right hand side $f$ in (15). The function $f$ is determined from the particle velocity $\frac{\partial u}{\partial \tau}$ on $\Gamma$, as supplied by the work group of W. Kropp at the Chalmers University in Gothenburg within the LeiStra3 cooperation [31]. See [32,33] and the references therein for details on their computations. These particle velocities are given by three complex values (amplitude and phase shift of the cosine function in each of the three space dimension) for 


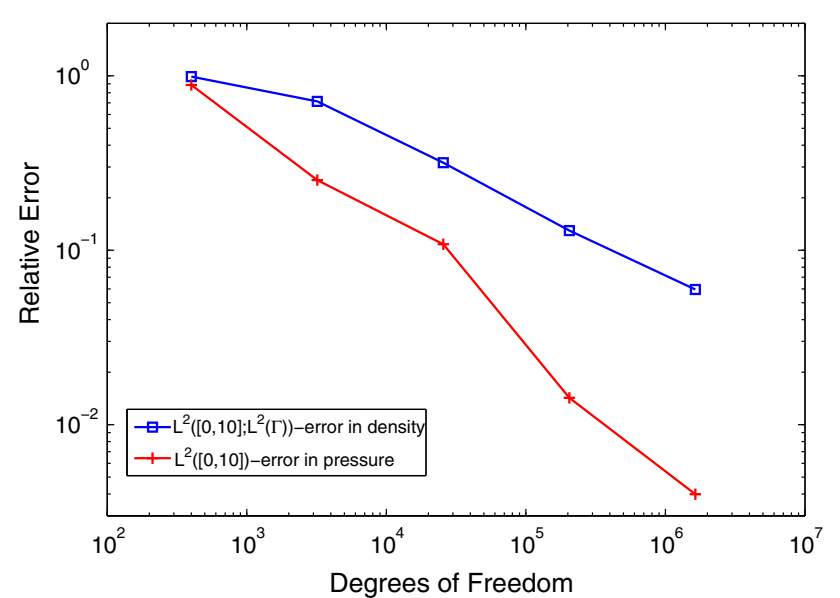

Fig. 2 Relative $L^{2}$-errors of density $\varphi_{\Delta t, h}$ and pressure $p_{\Delta t, h}$
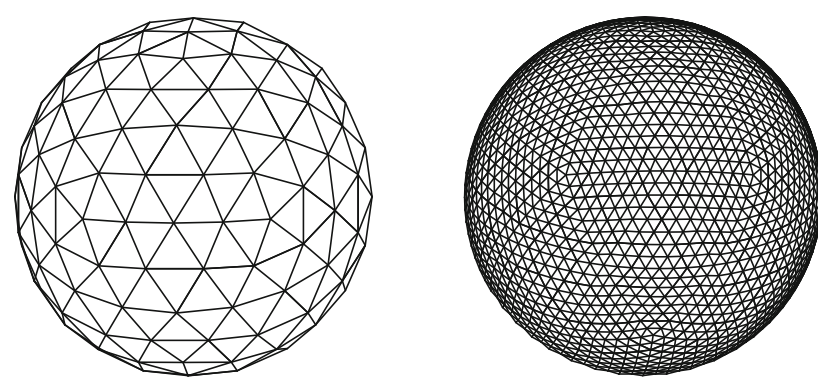

Fig. 3 Uniform meshes for the sphere with 320 and 5120 triangles

513 equidistant frequency points between $0 \mathrm{~Hz}$ and $1809.4 \mathrm{~Hz}$ in each of the 6027 nodes of the triangulation in the left part of Fig. 4. The tire at hand, including its rim, is a grown slick $205 / 55 \mathrm{R} 16$ at 2 bar pressure and was subject to $3415 \mathrm{~N}$ axle load at $50 \mathrm{~km} / \mathrm{h}$ on a street with an ISO 10844 surface. Due to the triangulation the normal is not continuous in the vertices and on the edges. Therefore, the particle velocities are interpolated piecewise linearly in space, such that their normal component can be evaluated in the interior of each triangle by the quadrature formula used to realize (15). According to the initial conditions (2b), the tire is initially not vibrating, and (2) describes the sound radiation of a transient tire. To obtain compatible boundary conditions, the time dependent particle velocity is multiplied with a $C^{2}$-continuous ramp up function $\phi(t)$ which is a (piecewise) polynomial of fifth degree satisfying $\phi(0)=\dot{\phi}(0)=\ddot{\phi}(0)=\dot{\phi}\left(t^{\prime}\right)=\ddot{\phi}\left(t^{\prime}\right)=0, \phi(t)=1$ for $t \geq t^{\prime}$. Here, $t^{\prime}$ denotes the length of the ramp up function and is chosen to be 0.3 in the numerical experiment. If $t^{\prime}$ is very small, the ramp is very steep, i.e. the supremum norm of its time derivative is large and thus requires smaller time steps. If $t^{\prime}$ is very large, the time domain, i.e. the number of time steps, is artificially enlarged.

Figure 5 shows the density of (7) for $\Delta t=0.01$ at different time steps for the tire $1 \mathrm{~mm}$ above the street. In addition to the known singularity in the cusp where the tire meets the
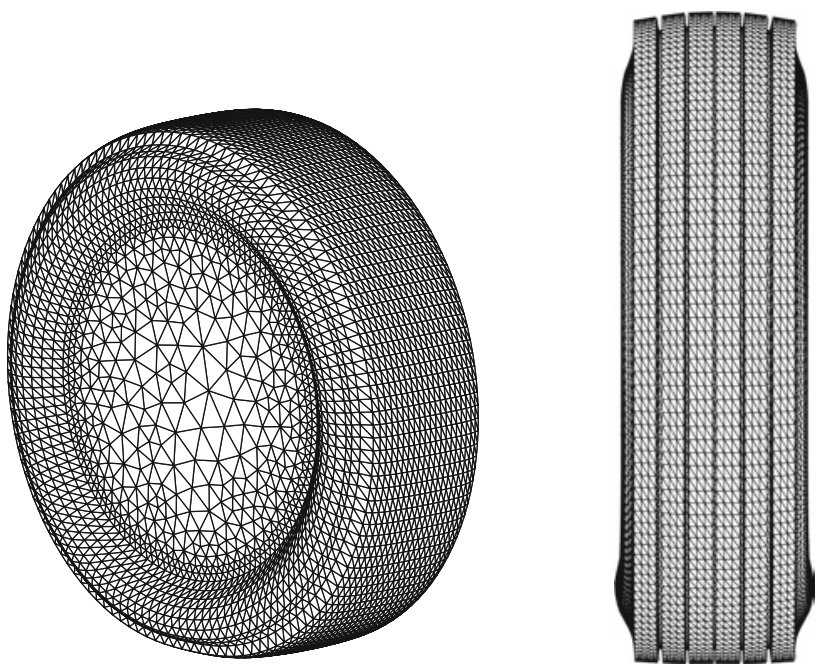

Fig. 4 Discretization of a 205/55R 16 passenger car tire (left) and a 315/80R22.5 rib truck tire (right) used for computation of tire vibration and Horn-effect

road a wave of large amplitude is observed which propagates around the tire. This motivates the study of a posteriori error estimates and adaptive discretizations for the solution of time domain boundary integral equations [34].

The A-weighted sound pressure level provides an approximation to the human perception of noise [32]. Figure 6a shows the A-weighted sound pressure level of the acoustic wave radiated from the discretized tire with $\Delta t=0.01$ averaged over 321 points in the hemisphere $\left\{x \in \mathbb{R}^{3}: x_{3} \geq\right.$ $\left.0,\|x\|_{2}=1\right\}$. The different curves have been obtained by a discrete fast Fourier transform of the calculated sound pressure level for times $\tau \geq \tau_{0}$, with $\tau_{0}=0,0.005$ and 0.02 . For comparison, Fig. 6b displays the same data but without Aweighting. The relative behavior did not change, but given the values of the A-weighting the slope decreases. For these figures, the time dependent sound pressure $p(t, x)=S \varphi(t, x)$, depicted for $x=(0.7071,0,0.7171)^{\top}$ in Fig. $7 \mathrm{a}$, is transformed into the frequency domain by the discrete FFT, Fig. 7b. A reference is given by the results of [31], the blue curve in Fig. 6, which was calculated by a BEM collocation method with piecewise constant trial functions [35] for the Helmholtz equation

$$
\begin{aligned}
& \Delta \hat{p}+\omega^{2} \hat{p}=0 \quad \text { in } \mathbb{R}^{+} \times \Omega, \\
& \frac{\partial \hat{p}}{\partial n}(\omega, x)=-\rho i \omega v_{n}(\omega, x) \text { in } \mathbb{R}^{+} \times \Gamma, \\
& \frac{\partial \hat{p}}{\partial x_{3}}(\omega, x)=0 \text { in } \mathbb{R}^{+} \times \Gamma_{\infty} .
\end{aligned}
$$

It is worth pointing out that for the blue curve a BurtonMiller stabilization is used for frequencies above $1000 \mathrm{~Hz}$. The remaining curves all result form the same TDBEM simulation where the difference is only the starting time from 


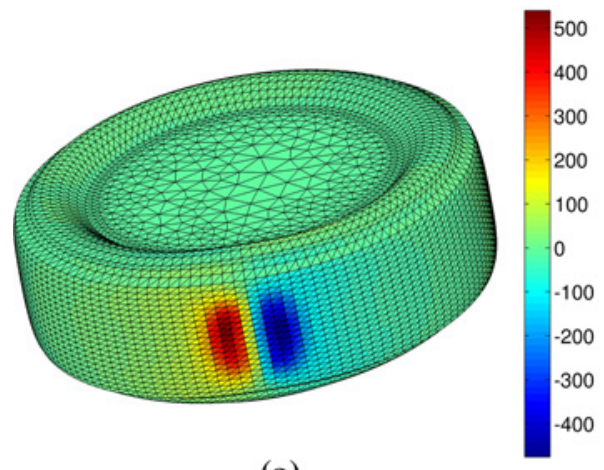

(a)

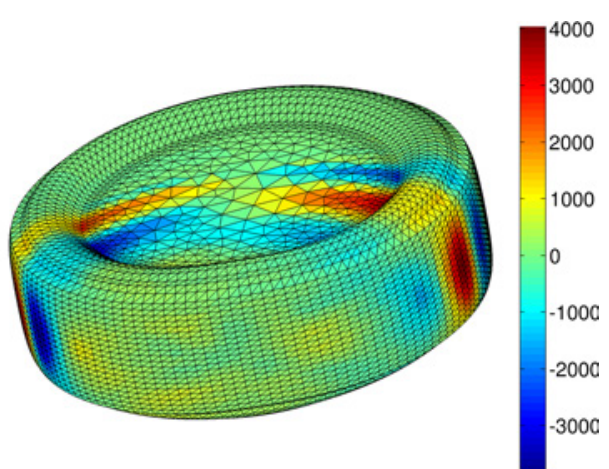

(b)

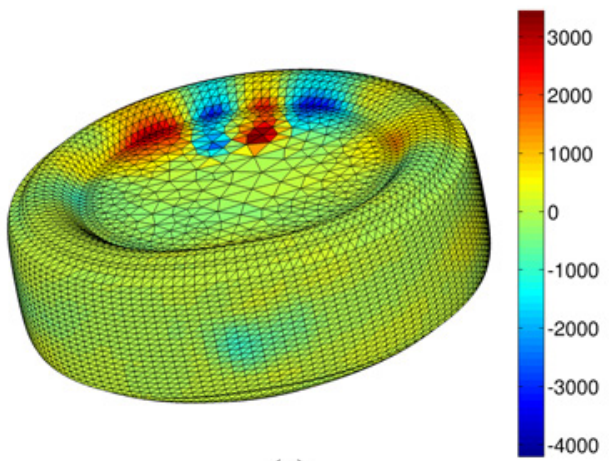

(c)

Fig. 5 Visualization of the density for vibrating tire for $\Delta t=0.01$, time step: 3 (a), 50 (b), 100 (c)

which onwards the point evaluation of the sound pressure is considered. Except for the green, diamond curve, the other ones have the same qualitative behavior and only differ significantly to the blue curve for the third-octave bands with center frequency 1600 and $2000 \mathrm{~Hz}$ (red, circle curve) or with center frequency 160 and $200 \mathrm{~Hz}$ (brown, square curve). Due to the use of the particle velocities from the frequency domain and the discretization of the ramp up function, a shock wave is emitted at the beginning of the simulation with the peaks for times less than $\tau=0.005 \mathrm{~s}$ in Fig. 7. Considering this artificial wave also in the Fourier transformation leads to distorted amplitudes in the frequency spectrum, hence the green diamond curve.

The simulation for the vibrating truck tire follows the same lines of code as the passenger car tire. The particle velocities are supplied for 457 equidistant frequency points between 0 and $2210.1 \mathrm{~Hz}$ in each of the 13034 nodes of the triangulation in the right part of Fig. 4. These data correspond to a grown $315 / 80 \mathrm{R} 22.5 \mathrm{rib}$ tire at $60 \mathrm{~km} / \mathrm{h}$ and 2000T axle load.

Figure 8 shows the $A$-weighted sound pressure level for the radiated sound pressure after $\tau \geq 0.04 \mathrm{~s}$, resp. $\tau \geq 0.05 \mathrm{~s}$, for the truck tire lifted $1 \mathrm{~mm}$ above the ground and time step size $\Delta t=0.25$ averaged over 321 points in the hemisphere $\left\{x \in \mathbb{R}^{3}: x_{3} \geq 0,\|x\|_{2}=2\right\}$. For comparison that figure also displays the measurements supplied within the LeiStra3 cooperation. The TDBEM simulation is capable of reproducing the qualitative characteristics of the sound emission with a peak between $500 \mathrm{~Hz}$ and $610 \mathrm{~Hz}$ and the general shape of the $A$-weighted sound pressure curve. For frequencies beyond $1000 \mathrm{~Hz}$ the details of the size of the time step and the transient behavior near time 0 crucially enter and motivate further study.

Compared to the car tire, simulations for the truck tire are complicated by trapped waves, which are reflected back and forth in the large treads and may build up to large amplitudes. These have to be resolved by fine discretizations to model phenomena like the Horn effect, which are dominated by the geometry of where the tire meets the road.

\section{Numerical experiments on the Horn effect}

The horn like geometry between the tire and the street is the reason for the amplification of a source close to the contact patch. The simulations use the geometrical setup as displayed in Fig. 9, see also [36].

The source emits the Dirac impulse

$G\left(t, x, y_{s r c}\right)=\frac{\delta\left(t-\left|x-y_{s r c}\right|\right)}{4 \pi\left|x-y_{s r c}\right|}+\frac{\delta\left(t-\left|x-y_{s r c}^{\prime}\right|\right)}{4 \pi\left|x_{s}-y_{s r c}^{\prime}\right|}$ 


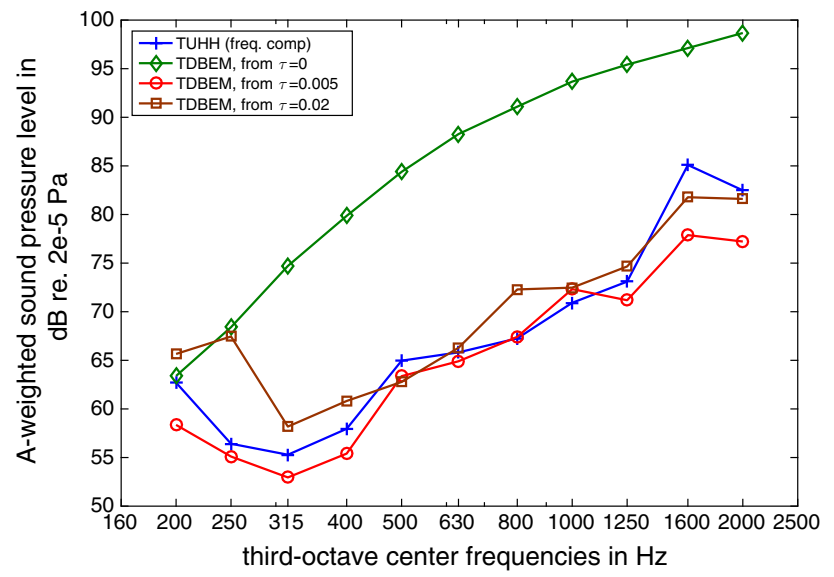

(a)

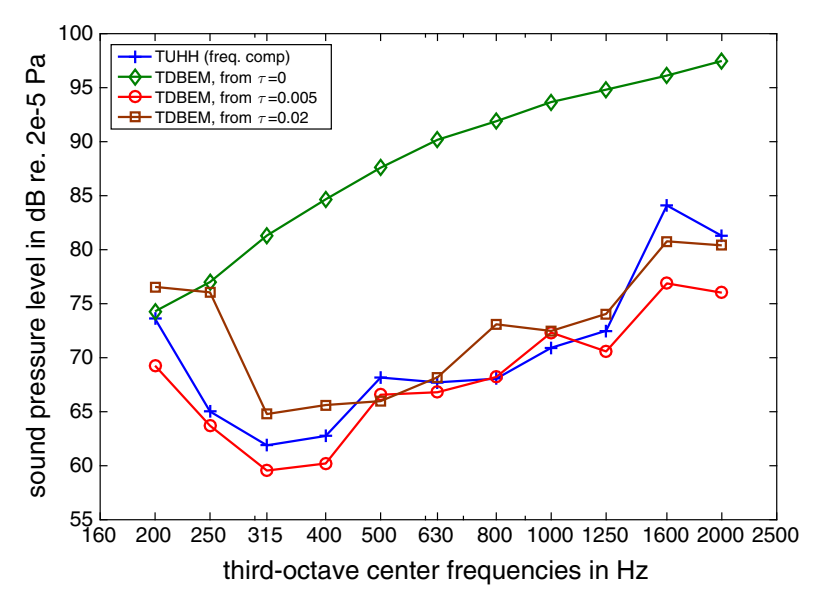

(b)

Fig. 6 Comparison of the sound pressure level averaged over 321 points and frequency bands for TDBEM and frequency domain BEM. With A-weighting (a), without A-weighting (b). Passenger car tire

to cover the Horn-effect for the entire frequency spectrum. Neglecting the tire vibrations $\rho c^{2} \frac{\partial^{2} u_{n}}{\partial t^{2}}$ in (2c) yields with $y_{s r c}=y_{s c r}^{\prime}=(0.08,0,0)^{\top}$ the right hand side $\left(\partial_{n} p^{I}=\right.$ $\left.\partial_{n} G\right)$

$$
\begin{aligned}
-2 & \int_{0}^{\infty} \int_{\Gamma} \frac{\partial p^{I}}{\partial n} \varphi_{j} \gamma^{n} d s_{x} d t=:(I)+(I I) \\
= & -4 \int_{I_{n}} \int_{\Gamma}\left\{\frac{n_{x}^{\top}\left(y_{s r c}-x\right)}{4 \pi\left|x-y_{s r c}\right|^{2}} \dot{\delta}\left(t-\left|x-y_{s r c}\right|\right)\right. \\
& \left.+\frac{n_{x}^{\top}\left(y_{s r c}-x\right)}{4 \pi\left|x-y_{s r c}\right|^{3}} \delta\left(t-\left|x-y_{s r c}\right|\right)\right\} \varphi_{j}(x) d s_{x} d t .
\end{aligned}
$$

Here,

$$
\begin{aligned}
(I I) & =-\int_{\Gamma \cap\left\{x \in \Gamma\left|t_{n-1} \leq\right| x-y_{s r c} \mid \leq t_{n}\right\}} \frac{n_{x}^{\top}\left(y_{s r c}-x\right)}{\pi\left|x-y_{s r c}\right|^{3}} \varphi_{j}(x) d s_{x} \\
& =-\int_{T_{j} \cap E\left(y_{s r c}\right)} \frac{n_{x}^{\top}\left(y_{s r c}-x\right)}{\pi\left|x-y_{s r c}\right|^{3}} d s_{x}
\end{aligned}
$$

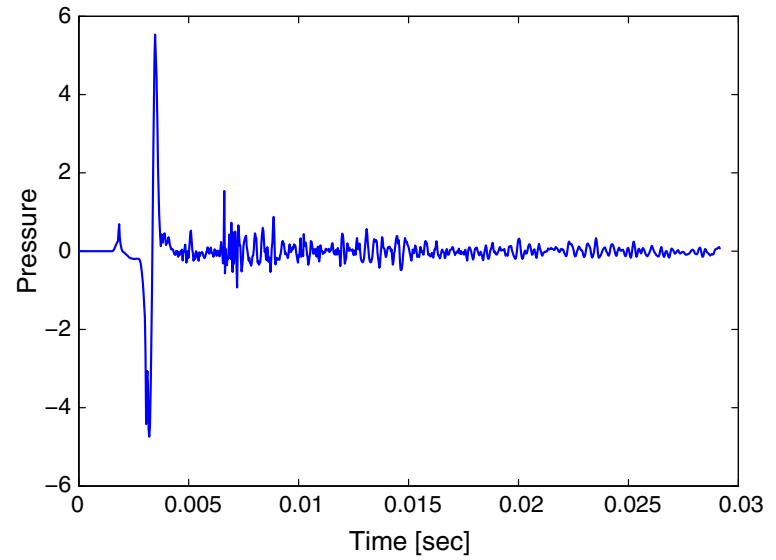

(a)

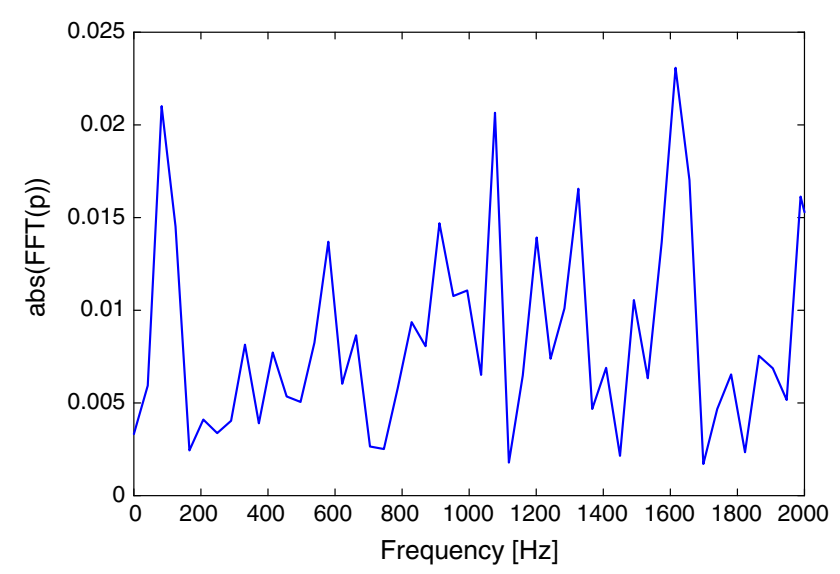

(b)

Fig. 7 Time dependent sound pressure $p=S \varphi$ and its FFT (for $\tau \geq$ $0.005)$ in $x=(0.7071,0,0.7171)^{\top}$ for $\Delta t=0.01$. Passenger car tire

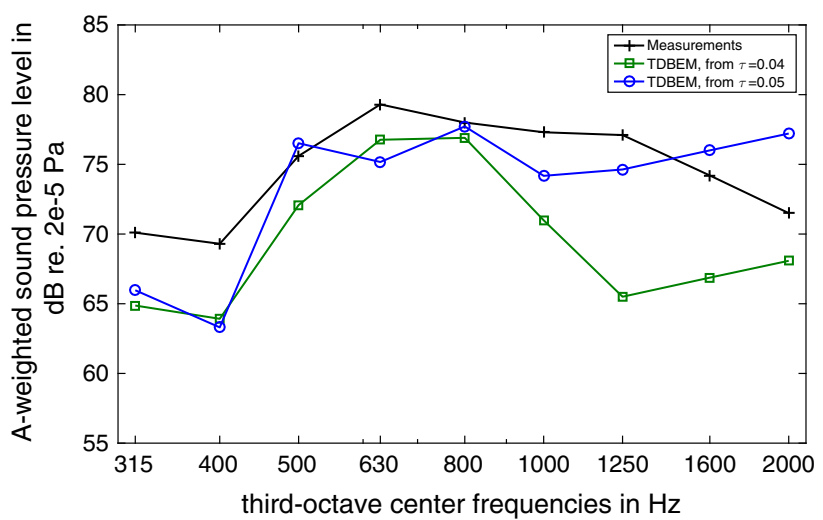

Fig. 8 A-weighted sound pressure level averaged over 321 points and frequency bands, $\Delta t=0.25$. Truck tire

is an integral over the domain of influence of $y_{s r c}$ and is computed in the same manner as the entries of the Galerkin matrix. The first term $(I)$ can be evaluated more explicitly by performing integration by parts in a distributional sense. 


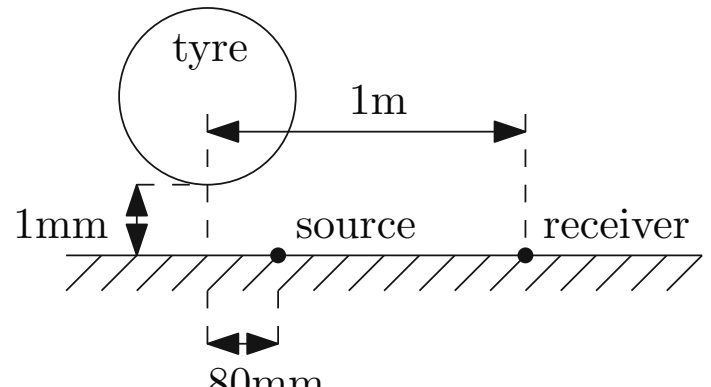

Fig. 9 Cross section of geometrical setup for Horn-effect, $x_{1} x_{3}$-plane, $x_{2}=0$

$$
\begin{aligned}
(I)= & 4 \int_{\mathbb{R}} \int_{\Gamma} \frac{n_{x}^{\top}\left(y_{s r c}-x\right)}{4 \pi\left|x-y_{s r c}\right|^{2}} \delta\left(t-\left|x-y_{s r c}\right|\right) \\
& \times\left\{-\delta\left(t_{n}\right)+\delta\left(t_{n-1}\right)\right\} \varphi_{j}(x) d s_{x} d t \\
= & \int_{T_{j}} \frac{n_{x}^{\top}\left(y_{s r c}-x\right)}{\pi\left|x-y_{s r c}\right|^{2}} \\
& \times\left\{-\delta\left(t_{n}-\left|x-y_{s r c}\right|\right)+\delta\left(t_{n-1}-\left|x-y_{s r c}\right|\right)\right\} d s_{x} \\
= & \int_{T_{j} \cap\left\{\left|x-y_{s r c}\right|=t_{n-1}\right\}} \frac{n_{x}^{\top}\left(y_{s r c}-x\right)}{\pi\left|x-y_{s r c}\right|^{2}} d s_{x} \\
& -\int_{T_{j} \cap\left\{\left|x-y_{s r c}\right|=t_{n}\right\}} \frac{n_{x}^{\top}\left(y_{s r c}-x\right)}{\pi\left|x-y_{s r c}\right|^{2}} d s_{x} \\
= & \frac{1}{\pi t_{n-1}^{2}} \int_{T_{j} \cap\left\{\left|x-y_{s r c}\right|=t_{n-1}\right\}} n_{x}^{\top}\left(y_{s r c}-x\right) d s_{x} \\
& -\frac{1}{\pi t_{n}^{2}} \int_{T_{j} \cap\left\{\left|x-y_{s r c}\right|=t_{n}\right\}} n_{x}^{\top}\left(y_{s r c}-x\right) d s_{x} \\
= & n_{x}^{\top}\left(y_{s r c}-x\right)\left\{\frac{\zeta\left(t_{n-1}\right)}{\pi t_{n-1}^{2}}-\frac{\zeta\left(t_{n}\right)}{\pi t_{n}^{2}}\right\} \quad \forall x \in T_{j}
\end{aligned}
$$

where $\zeta(t)$ denotes the length of the curve $T_{j} \cap\left\{\left|x-y_{s r c}\right|=\right.$ $t$ \}. Note that the integrand $n_{x}^{\top}\left(y_{s r c}-x\right)$ is constant on each triangle $T_{j}$. Indeed, if $x, y \in T_{j}$, then $x-y \perp n_{x}$ and, therefore,

$n_{x}^{\top}\left(y_{s r c}-x\right)=n_{x}^{\top}\left(y_{s r c}-y-(x-y)\right)=n_{x}^{\top}\left(y_{s r c}-y\right)$.

Therewith, the density can be computed with the MOT Algorithm 1 and the time dependent sound pressure in the receiver by point evaluations of the single layer potential. The frequency dependent amplification in the receiver point $x_{f p}=(1,0,0)^{\top}$ can be expressed as, see e.g. [36, Eq. 7],

$$
\Delta L_{H}(\omega)=20 \log _{10}\left(\frac{\left|\hat{p}_{1}\left(\omega, x_{f p}\right)\right|}{\left|\hat{p}_{2}\left(\omega, x_{f p}\right)\right|}\right)
$$

where $\hat{p}_{2}$ is the Fourier transformed sound pressure of the emitted Dirac impulse and $\hat{p}_{1}$ the Fourier transformed sound pressure of the Dirac impulse overlaid with the sound pressure of the tire. The Fourier transformations are realized by

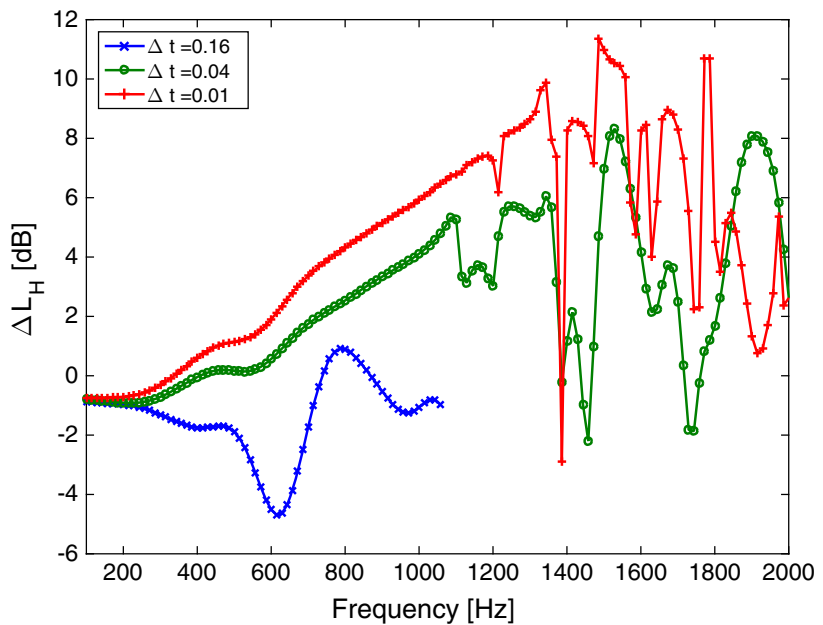

Fig. 10 Amplification due to Horn-effect, slick 205/55R16 tire 1mm above ground

the discrete FFT applied to a sample of each wave where the time step size is the same as for the computation of the density. For the sampling of the Dirac impulse the function $\delta\left(t-\left|x_{f p}-y_{s r c}\right|\right)$ is first approximated by a rectangular function where the rectangle has a width of $\Delta t$, center $\left|x_{f p}-y_{s c r}\right|$ and height $(\Delta t)^{-1}$.

Figure 10 displays the computed amplifications for the frequencies from 200 to $2000 \mathrm{~Hz}$ for the tire from Sect. 6, c.f. Fig. 4. The total time length is $T=24$ and only the time step size $\Delta t$ varies between $0.16,0.04$ and 0.01 . Figure 10 shows that there is a maximal time step length which should not be exceeded as the results are dominated by the numerical approximation error, c.f. curve for $\Delta t=0.16$. For smaller time step sizes, $\Delta t=0.04, \Delta t=0.01$, the amplification curves have similar characteristics, but the absolute level still depends significantly on the time step size. For smaller time step sizes more reflections in the horn can be resolved, but these are responsible for the sound amplification.

\section{Moving and rolling tires and the Doppler effect}

In light of Sect. 2 an acoustic point source $q(t, x)$ is considered, which moves with constant velocity $v$ along the $x_{1}$-axis at height $h$ above the $x_{1} x_{2}$-plane in the time scaled situation of (2), i.e. $\mathbf{v}=(v, 0,0)^{\top}$. The acoustic sound pressure $p$ is measured at $y=\left(y_{1}, y_{2}, y_{3}\right)$. The Lorentz transformation with respect to $x$ and $t$ is

$\tilde{t}=\gamma(t-v x), \quad \tilde{x}_{1}=\gamma\left(x_{1}-v t\right), \quad \tilde{x}_{2}=x_{2}, \quad \tilde{x}_{3}=x_{3}$,

where $\gamma=\frac{1}{\sqrt{1-v^{2}}}$. Applying this transformation to the wave equation (2a) leads to

$\frac{\partial^{2} u_{L}}{\partial \tilde{t}^{2}}-\Delta_{L} u_{L}=0$. 
Here, $u_{L}$ is the unknown and $\Delta_{L}$ is the Laplace operator written in the new coordinates $\left(\tilde{x}_{1}, \tilde{x}_{2}, \tilde{x}_{3}\right)$. The Green's function, which describes a moving point source in the new coordinates, is given by

$G(\tilde{x}, \tilde{t} ; \tilde{y}, \tilde{\tau})=\frac{\delta(\tilde{t}-\tilde{\tau}-\tilde{R})}{4 \pi \tilde{R}\left[1-M_{\tilde{R}}\right]}$,

where $M_{\tilde{R}}=\frac{\mathbf{v}^{\top}(\tilde{x}-\tilde{y})}{\tilde{R}}, \tilde{R}=|\tilde{x}-\tilde{y}|$ and $M_{\tilde{R}^{\prime}}=\frac{\mathbf{v}^{\top}\left(\tilde{x}-\tilde{y}^{\prime}\right)}{\tilde{R}^{\prime}}$, $\tilde{R}^{\prime}=\left|\tilde{x}-\tilde{y}^{\prime}\right|$. The Lorentz transformation allows the moving source to be treated as a stationary source in the moving frame, and provides a model for the motion of the source. The Dirichlet and Neumann boundary conditions are preserved under this transformation and one obtains the corresponding half-space Green's function

$G(\tilde{x}, \tilde{t} ; \tilde{y}, \tilde{\tau})=\frac{\delta(\tilde{t}-\tilde{\tau}-\tilde{R})}{4 \pi \tilde{R}\left[1-M_{\tilde{R}}\right]}+\frac{\delta\left(\tilde{t}-\tilde{\tau}-\tilde{R}^{\prime}\right)}{4 \pi \tilde{R}^{\prime}\left[1-M_{\tilde{R}^{\prime}}\right]}$

for Neumann boundary conditions on $\Gamma_{\infty}$. As before, the single layer potential ansatz

$$
\begin{aligned}
p(\tilde{t}, \tilde{x})= & S \varphi(\tilde{t}, \tilde{x})=\int_{\Gamma} \frac{\varphi(\tilde{t}-\tilde{R}, y)}{4 \pi \tilde{R}\left[1-M_{\tilde{R}}\right]} d s_{y} \\
& +\int_{\Gamma} \frac{\varphi\left(\tilde{t}-\tilde{R}^{\prime}, y\right)}{4 \pi \tilde{R}^{\prime}\left[1-M_{\tilde{R}^{\prime}}\right]} d s_{y}
\end{aligned}
$$

for the Neumann problem leads to a time domain boundary integral equation

$$
\left(-I+K^{\prime}\right) \varphi(\tilde{t}, \tilde{x})=2 \frac{\partial p}{\partial n}(\tilde{t}, \tilde{x})
$$

Here, the time domain adjoint double layer operator in the moving frame is given by

$$
\begin{aligned}
K^{\prime} \varphi(\tilde{t}, \tilde{x})= & \frac{1}{2 \pi} \int_{\Gamma} \frac{n_{x}^{\top}(\tilde{y}-\tilde{x})}{\tilde{R}\left[1-M_{\tilde{R}}\right]} \dot{\varphi}(\tilde{t}-\tilde{R}, y) d s_{y} \\
& +\frac{n_{x}^{\top}(\tilde{x}-\tilde{y})+\tilde{R} n_{x}^{\top} v}{\tilde{R}\left[1-M_{\tilde{R}}\right]^{2}} \varphi(\tilde{t}-\tilde{R}, y) d s_{y} \\
& +\frac{1}{2 \pi} \int_{\Gamma} \frac{n_{x}^{\top}\left(\tilde{x}-\tilde{y}^{\prime}\right)}{\tilde{R}^{\prime}\left[1-M_{\tilde{R}}\right]} \dot{\varphi}\left(\tilde{t}-\tilde{R}^{\prime}, y\right) d s_{y} \\
& +\frac{n_{x}^{\top}\left(\tilde{x}-\tilde{y}^{\prime}\right)+\tilde{R}^{\prime} n_{x}^{\top} v}{\tilde{R}^{\prime}\left[1-M_{\tilde{R}}\right]^{2}} \varphi(\tilde{t}-\tilde{R}, y) d s_{y} .
\end{aligned}
$$

The discretization technique for (25) is the same as described in Sect. 2.

In the following numerical experiment for the Doppler effect, $v=0.02$ is chosen in units where $c=1$, i.e. the velocity is $6.86 \mathrm{~ms}^{-1}$ for $c=343 \mathrm{~ms}^{-1}$, for the unit sphere

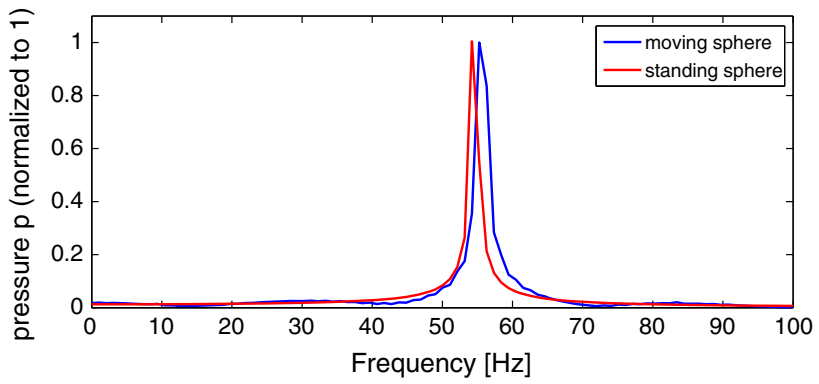

Fig. 11 Doppler effect: Fourier transformed sound pressure versus sinusoidal signal as a function of frequency in the point $x_{R}=(13,0,0)$

which is $0.001 \mathrm{~m}$ above the plane. The sphere radiates at $f_{S}=(2 \pi)^{-1} \cdot 343 \mathrm{~Hz} \approx 54.59 \mathrm{~Hz}$. The theory of the Doppler effect predicts an observed frequency of $f_{R}=$ $c(c-v)^{-1} f_{S} \approx 55.70 \mathrm{~Hz}$ in the point $x_{R}=(13,0,0)^{\top}$, neglecting the angle changes between the receiver and sender as it moves towards the receiver. For the computations 320 uniform triangles are used for the space discretization, $\Delta t=$ 0.15 , i.e. $\Delta \tau \approx 4.37 \cdot 10^{-4} s$ and 2194 time steps. Figure 11 shows the discrete Fourier transformed sound pressure $p\left(f, x_{R}\right)$ (normalized to 1 ) in $x_{R}$ for frequencies up to $100 \mathrm{~Hz}$ and compares the signal with the discrete Fourier transform of a sinusoidal wave $\sin \left(2 \pi f_{s} \tau_{j}\right)$ in the same time nodes $\tau_{j}=(j-1) \Delta \tau$. The expected frequency shift of $2 \%$ is clearly resolved by the numerical computations.

As a variation of the moving tire, the following idealized model of a rolling tire is considered:

In addition to moving velocity $v$ along $x_{1}$, it rotates around its center with an angular velocity $\omega$ around the $x_{2}$-axis through $x_{3}=R+h$ determined by the rolling condition $\omega=\frac{v}{R}, R$ the radius of the tire. As the tire is rotating, but not the street, one cannot use a rotating fundamental solution of the wave equation, but rather rotates the data $f$ on the tire. Physically, this accounts for the rotation of the displacement $u_{n}$, but not for how it is affected by the contact with the street.

Given data $f=\left(t, x_{1}, x_{2}, x_{3}\right)$ in the stationary frame, data in the rotating frame $\left(\widetilde{x}_{1}, \widetilde{x}_{2}, \widetilde{x}_{3}\right)$ are obtained as:

$$
\left(\begin{array}{l}
x_{1}-\omega R t \\
x_{2} \\
x_{3}-R-h
\end{array}\right)=\left(\begin{array}{lll}
\cos (\omega t) & 0 & \sin (\omega t) \\
0 & 1 & 0 \\
-\sin (\omega t) & 0 & \cos (\omega t)
\end{array}\right)\left(\begin{array}{l}
\widetilde{x}_{1} \\
\widetilde{x}_{2} \\
\widetilde{x}_{3}-R-h
\end{array}\right) .
$$

The data on the rolling tire are hence given as

$$
\begin{aligned}
& f\left(t, \cos (\omega t) \widetilde{x}_{1}+\omega R t+\sin (\omega t)\left(\widetilde{x}_{3}-h\right), x_{2}, R\right. \\
& \left.\quad+h-\sin (\omega t) \widetilde{x}_{1}+\cos (\omega t)\left(\widetilde{x}_{3}-R-h\right)\right) .
\end{aligned}
$$

Figure 12 shows the computational results for a rolling unit sphere. A monopole sound source is placed at the front of the sphere, which moves with a constant velocity $v=24.6 \mathrm{~km} / \mathrm{h}$ above the plane with $h=0.001 \mathrm{~m}$. The sphere is approximated by an icosahedron with 320 elements, and $\Delta \tau=$ 


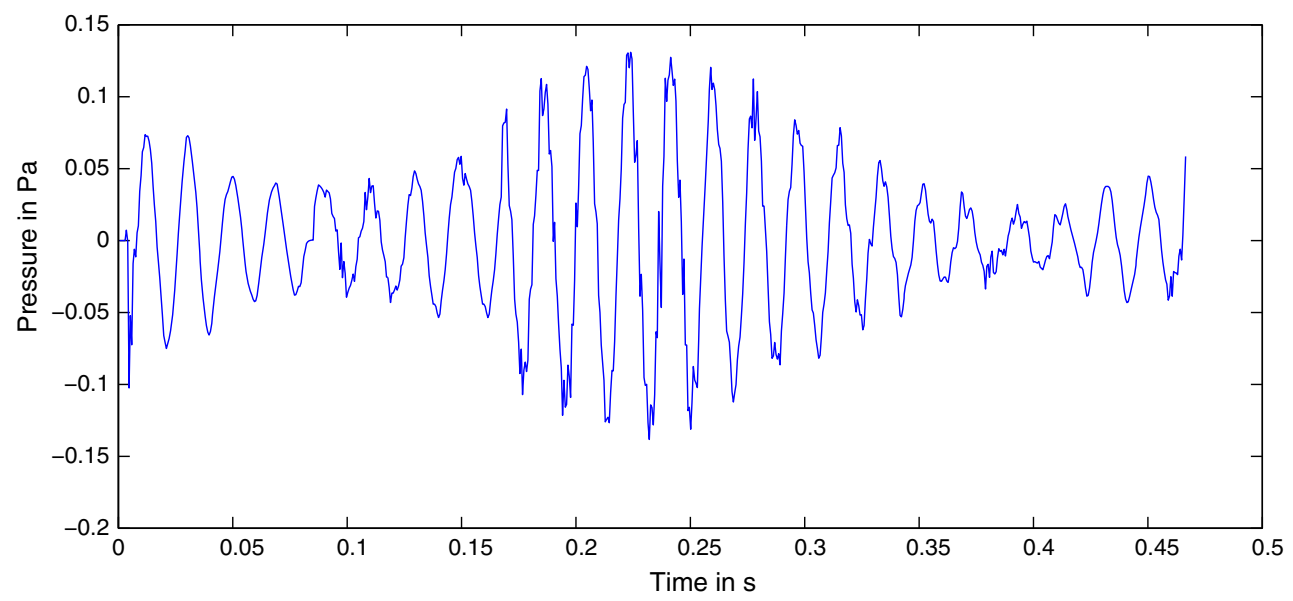

Fig. 12 Rolling tire: Sound pressure (in Pa) at an evaluation point $1.5 \mathrm{~m}$ away from the road for $343 \mathrm{~Hz}$

$0.001431 s$. The sound pressure is evaluated at $(2 m, 0,0)^{\top}$, and the sphere rolls over this point at time $\tau=0.25 \mathrm{~s}$. A highfrequency numerical artifact, wriggles, are superimposed on the actual sound pressure.

\section{Conclusions}

This work presents a practically stable and accurate time domain Galerkin boundary element method based on a time-dependent integral equation of the second kind. After validating the approach in an example problem, numerical experiments are presented for benchmarks from traffic noise. They underline the suitability of the scheme for practical engineering problems in the sound radiation of tires, including the singular geometries of the horn effect and the treads of a truck tire, as well as moving and rolling sources (Doppler effect). In particular, a qualitative agreement between simulations and experiments is observed, and a quantitative agreement with frequency domain computations.

Using a Fast Fourier Transform, the approach also proves competitive with frequency domain boundary elements when a broad band of frequencies is involved. Furthermore, the time domain formulation applies to problems beyond the range of application of frequency domain methods, such as rolling sources and time-dependent domains. Future work will particularly consider nonlinear problems that cannot be formulated in the frequency domain.

Acknowledgments Zouhair Nezhi was funded by BMWi under the project SPERoN 2020, part II, Leiser Straßenverkehr, Grant number 19 U 10016 F. Heiko Gimperlein acknowledges partial support by the Danish National Research Foundation (DNRF) through the Centre for Symmetry and Deformation, the Danish Science Foundation (FNU) research Grant 10-082866 and the ERC Advanced Grant HARG 268105.

\section{References}

1. Ding Y, Forestier A, Duong TH (1989) A Galerkin scheme for the time domain integral equation of acoustic scattering from a hard surface. J Acoust Soc Am 86(4):1566-1572

2. Friedmann M, Shaw R (1962) Diffraction of pulses by cylindrical obstacles of arbitrary cross section. J Appl Mech 29:40-46

3. Cruse TA, Rizzo FJ (1968) A direct formulation and numerical solution of the general transient elastodynamic problem I. J Math Anal Appl 22:244-259

4. Mansur WJ (1983) A time-stepping technique to solve wave propagation problems using the boundary element method. Ph.D. thesis, University of Southampton

5. Bamberger A, Ha-Duong T (1986) Formulation variationnelle espace-temps pour le calcul par potentiel retarde de la diffraction d'une onde acoustique. Math Methods Appl Sci 8:405-435

6. Terrasse I (1993) Résolution mathématique et numérique des équations de Maxwell instationnaires par une méthode de potentiels retardés. Ph.D. thesis, École Polytechnique

7. Ha-Duong T, Ludwig B, Terrasse I (2003) A Galerkin BEM for transient acoustic scattering by an absorbing obstacle. Int J Numer Methods Eng 57(13):1845-1882

8. Maischak M, Ostermann E, Stephan EP (2009) TD-BEM for sound radiation in three dimensions and the numerical evaluation of retarded potentials. In: Proceedings of the NAG/DAGA 2009 international conference on acoustics. Rotterdam, pp 633-636

9. Stephan EP, Maischak M, Ostermann E (2008) Transient boundary element method and numerical evaluation of retarded potentials. In: Computational science-ICCS 2008, pp 321-330. Springer, Berlin

10. Ostermann E (2009) Numerical methods for space-time variational formulations of retarded potential boundary integral equations. $\mathrm{Ph} . \mathrm{D}$. thesis, Leibniz Universität Hannover

11. Banjai L, Sauter S (2008/2009) Rapid solution of the wave equation in unbounded domains. SIAM J Numer Anal 47:227-249

12. Banjai L, Schanz M (2012) Wave propagation problems treated with convolution quadrature and bem. In: Fast boundary element methods in engineering and industrial applications. Lecture notes in applied and computational mechanics, vol 63. Springer, Berlin, pp 145-184

13. Ochmann M (2011) Closed form solutions for the acoustical impulse response over a masslike or an absorbing plane. J Acoust Soc Am 129(6):3502-3512 
14. Stütz M, Ochmann M (2008) Stability behaviour and results of a transient boundary element method for exterior radiation problems. Acoustics08-Paris

15. Stütz M, Ochmann M (2009) Simulation of transient sound radiation using the time domain boundary element method. In: Proceedings of the NAG/DAGA 2009 international conference on acoustics. Rotterdam, pp 629-632

16. Antes H, Baaran $\mathbf{J}$ (2001) Noise radiation from moving surfaces. Eng Anal Bound Elem 25:725-740

17. Gennaretti M, Morino L (1992) A boundary element method for the potential, compressible aerodynamics of bodies in arbitrary motion. Aeronaut J 96:15-19

18. Rasmussen KM, Nielsen SRK, Kirkegaard PH (2001) Boundary element method solution in the time domain for a moving timedependent force. Comput Struct 79:691-701

19. Hatzigeorgiou GD, Beskos DE (2011) Dynamic inelastic structural analysis by the bem: a review. Eng Anal Bound Elem 35:159-169

20. Stavroulakis GE, Antes H, Panagiotopoulos PD (1999) Transient elastodynamics around cracks including contact and friction. Comput Methods Appl Mech Eng 177:427-440

21. Abboud T, Joly P, Rodriguez J, Terrasse I (2011) Coupling discontinuous Galerkin methods and retarded potentials for transient wave propagation on unbounded domains. J Comput Phys 230(15):5877-5907

22. Banjai L, Kachanovska M (2014) Fast convolution quadrature for the wave equation in three dimensions. J Comput Phys 279:103126

23. Sylvand G (2002) La methode multipole rapide en electromagnetisme: performances, parallelisation, applications. Ph.D. thesis, Ecole Nationale des Ponts et Chaussees

24. Costabel M (2004) Time-dependent problems with the boundary integral equation method. Encyclopedia of computational mechanics 1 , Ch 25

25. Ha-Duong T (2003) On retarded potential boundary integral equations and their discretisation. In: Ainsworth M, Davies P, Duncan DB, Martin PA, Rynne B (eds) Topics in computational wave propagation. Springer, Berlin, pp 301-336
26. Sayas FJ (2013) Retarded potentials and time domain boundary integral equations: a road-map. Lecture notes

27. Gimperlein H, Nezhi Z, Stephan EP (2015) A priori error estimates for a time-dependent boundary element method for the acoustic wave equation in a half-space. Math Methods Appl Sci doi:10. 1002/mma.3340

28. Epstein CL, Greengard L, Hagstrom T (2015) On the stability of time-domain integral equations for acoustic wave propagation. Preprint

29. Nezhi Z (2014) Adaptive time domain boundary element method for sound radiation of tyres. Ph.D. thesis, Leibniz Universität Hannover

30. Veit A (2011) Numerical methods for the time-domain boundary integral equations. Ph.D. thesis, Universität Zürich

31. von Estorff Oet al (2014) Abschlussbericht SPERoN 2020-Teil II, Entwicklung eines performanten Rechenmodells zur Berechnung und Reduzierung der Geräuschabstrahlung von Reifen

32. Hoever C (2012) The influence of modelling parameters on the simulation of car tyre rolling losses and rolling noise. $\mathrm{Ph} . \mathrm{D}$. thesis, Chalmers University of Technology

33. Kropp W, Sabiniarz P, Brick H, Beckenbauer T (2012) On the sound radiation of a rolling tyre. J Sound Vib 331(8):1789-1805

34. Gimperlein H, Nehzi Z, Stephan EP (2015) A residual a posteriori error estimate for the time-domain boundary element method. In preparation

35. Keuchel S, Gehlken M, von Estorff O (2012) Halfspace formulations for the boundary element method in 3D-acoustics using the fast multipole method. In: Proceedings of the 19th international congress on sound and vibration

36. Kropp W, Bécot FX, Barrelet S (2000) On the sound radiation from tyres. Acta Acust United Acust 86(5):769-779 